\title{
An OGLE view of the bulge and Sagittarius
}

\author{
Shoko Jin ${ }^{1}$, Eva K. Grebel ${ }^{2}$ and Raoul Haschke ${ }^{2}$ \\ ${ }^{1}$ Kapteyn Astronomical Institute, University of Groningen, \\ P.O. Box 800, 9700 AV Groningen, the Netherlands \\ email: jin@astro.rug.nl \\ ${ }^{2}$ ARI, ZAH, University of Heidelberg, Mönchhofstr. 12-14, 69120 Heidelberg, Germany
}

\begin{abstract}
We use observations of OGLE-III ab-type RRLyrae stars towards the Galactic centre (Soszyński et al. 2011, AcA 6,1) to study the metallicity and 3D spatial distribution of the old and metal-poor component of the Galactic bulge. Metallicities and distances to the RRLyrae stars are derived photometrically from Fourier-component analyses of their light curves, allowing the determination of distances accurate to $\sim 7 \%$ from 0 to $40 \mathrm{kpc}$. The distance distribution of the RRLyrae stars peaks at $8.8 \mathrm{kpc}$, with the data indicating the presence of a bar-like structure inclined at $\sim 30^{\circ}$ to the line of sight. The dataset also exhibits a secondary concentration of stars beyond the Galactic centre at $\sim 27 \mathrm{kpc}$. This is consistent with the distance to the Sagittarius (Sgr) dwarf galaxy, and can be attributed to the serendipitous alignment of the Sgr stream with the bulge. This dataset allows the Sgr stream to be traced in this part of the sky for the first time. The underlying metallicity distributions are determined to have mean \pm intrinsic width of $[\mathrm{Fe} / \mathrm{H}]_{\text {bulge }}=-1.24 \pm 0.23$ dex and $[\mathrm{Fe} / \mathrm{H}]_{\mathrm{Sgr}}=-1.53 \pm 0.14 \mathrm{dex}$.
\end{abstract}

Keywords. Galaxy: bulge, galaxies: individual (Sagittarius)
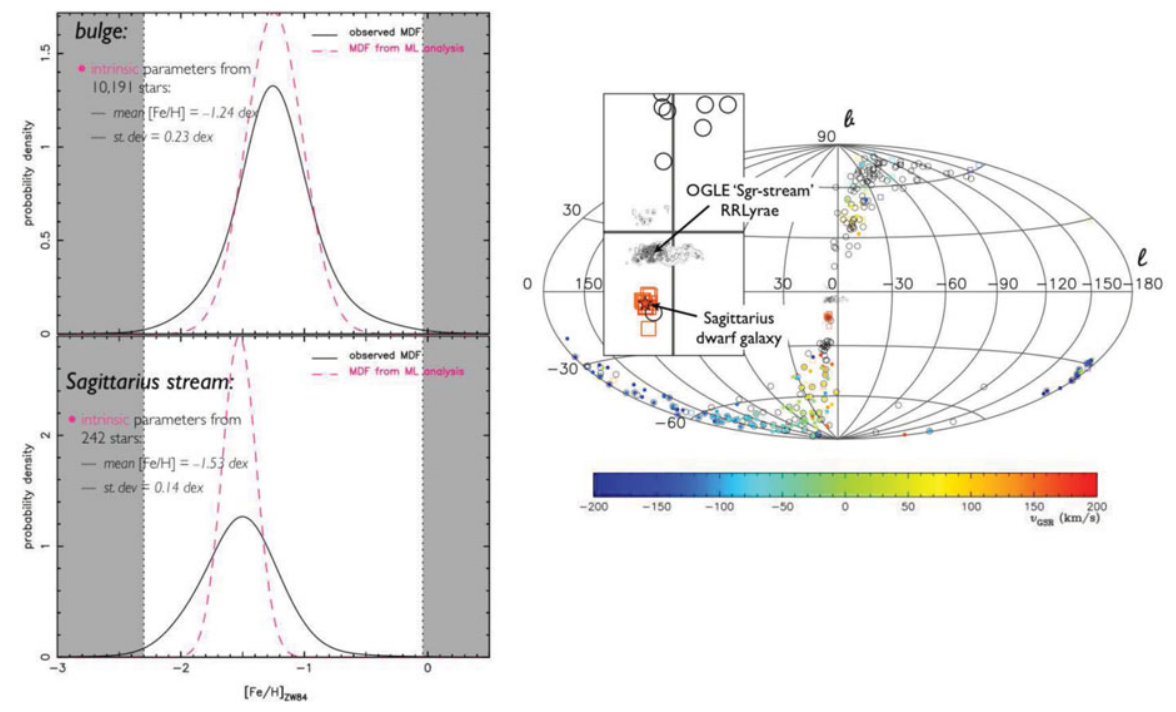

Figure 1. Left: observed and intrinsic metallicity distribution functions (MDFs) for the bulge and Sgr stream. Intrinsic MDFs of the underlying stellar population are derived from a maximum-likelihood (ML) analysis, assuming a Gaussian model for the underlying distribution. Photometric metallicities are on the Zinn \& West scale (1984, ApJ 55, 45). Right: likely Sgr-stream member RRLyrae stars from OGLE (shown as a contour map in number density) in the context of an all-sky view of the Sgr stream as traced by 2MASS M giants (circles, Majewski et al. 2004, AJ 128, 245) and carbon stars (squares, Totten \& Irwin 1998, MNRAS 294, 1; Ibata et al. 2001, ApJ 551, 294). Colours denote line-of-sight velocities with respect to the Galactic Standard of Rest, where available. 